\title{
Periodic Magnetization Pattern for Controlled Domain Wall Motion in Nanowires.
}

Sergei Lopatin ${ }^{1}$, Yurii P. Ivanov ${ }^{1}$, Jurgen $\operatorname{Kosel}^{1}$ and Andrey Chuvilin ${ }^{2,3}$.

${ }^{1}$ King Abdullah University of Science \& Technology, Thuwal, Makkah 23955, Saudi Arabia

2. CIC nanoGUNE, Av. de Tolosa 76, E-20018, Donostia-San Sebastian, Spain

3. IKERBASQUE, Basque Foundation for Science, Maria Diaz de Haro 3, 48013 Bilbao, Spain.

Cylindrical magnetic nanowires (NWs) are attractive materials for the next generation of 3D data storage devices [1], due to potentially very high speed of domain wall (DW) motion and their efficient fabrication in dense arrays. Well-defined pinning sites (i.e. energy potentials) are required to obtain a reliable control over DW motion along the NW [2]. Up until now such control has not been shown.

In this study, we introduce a novel approach based on cylindrical NWs with periodic variation of $\mathrm{Ni}(\mathrm{fcc})$ and $\mathrm{Co}(\mathrm{hcp})$ segments, where the interfaces between segments act as a reliable pinning sites for DWs. Single crystal hcp Co NWs have an easy axis, defined by the strong magnetocrystalline anisotropy, perpendicular to the NW [3]. In contrast, Ni NWs have the easy axis parallel to the NW, due to the magnetocrystalline anisotropy being weaker than the shape anisotropy [4]. As a result, the directions of the magnetic anisotropies in adjacent segments of $\mathrm{Ni} / \mathrm{Co} \mathrm{NWs}$ are perpendicular to each other.

The domain wall pinning in our Ni/Co NWs is comprehensively studied by a combination of magnetic force microscopy (MFM) and several transmission electron microscopy (TEM) techniques dedicated to magnetic samples. These techniques include electron holography and recently developed modification of differential phase contrast, based on the idea of virtual bright field unitary detector (VBF-DPC) [5].

Images for the VBF-DPC were acquired on a Titan 60-300 TEM (FEI Co) equipped with a high brightness electron gun (x-FEG), and Fischione HAADF STEM detector. The microscope was operated at $200 \mathrm{keV}$ acceleration voltage with objective lens set to $0 \%$ to allow zero magnetic field environment.

Multisegment $\mathrm{Co} / \mathrm{Ni} \mathrm{NWs}$ were prepared by the electrodeposition of $\mathrm{Co}$ and $\mathrm{Ni}$ into anodic alumina membranes by using two different aqueous electrolytes. The electroplating conditions for the Co segments were chosen to yield a magnetocrystalline anisotropy in-plane perpendicular to the NW. The length of the segments $300 \mathrm{~nm}-10$ um was controlled by deposition time. Our TEM study showed that both $\mathrm{Co}$ and Ni segments grow homogeneously with a constant speed inside a membrane and have sharp atomic interface (HR-TEM, EELS).

We observed strong periodic stray fields following the segmentation of NWs (VBF-DPC, MFM), which were correlated with the positions of the interfaces. These stray fields were able to effectively pin the 3D DW during magnetization switching as well as after local DW nucleation introduced by MFM tip. A detailed analysis of the magnetic structure of the DW obtained in electron holography (Fig.1a,b) and VBF-DPC (Fig.2a) revealed a vortex type DW with a Bloch point on the axis (center) of the NW. This finding is in a good agreement with finite element micromagnetic simulations (Fig.2b).

In conclusion, by comprehensive study with various electron microscopy techniques we demonstrate that $\mathrm{Ni} / \mathrm{Co}$ cylindrical nanowires with periodic magnetization pattern are promising candidates for realization of novel 3D memory devices based on propagation of DWs along the NW. 
References:

[1] S. S. P. Parkin et al, Science 320 (2008), p.190.

[2] Yu. P. Ivanov et al, J. Phys. D: Appl. Phys. 46 (2013), p. 485001.

[3] Yu. P. Ivanov et al, Nanotechnology 25 (2014), p. 475702.

[4] E. V. Vidal et al, Appl. Phys. Lett. 106 (2015), p. 032403.

[5] S. Lopatin et al, Ultramicroscopy 162, (2016), p. 74.
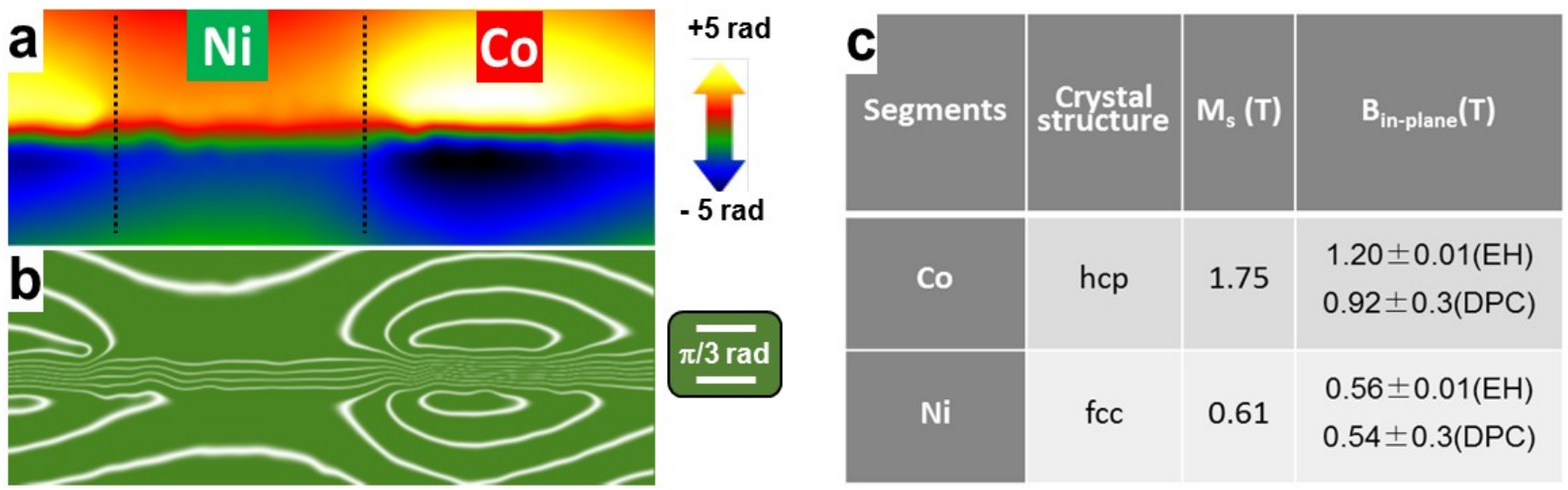

Figure 1. EH analysis of $\mathrm{Ni} / \mathrm{Co}$ nanowire. a, Reconstructed from the electron hologram magnetic phase gradient. b, In-plane magnetic induction map. c, The comparison between bulk values of the Co and $\mathrm{Ni}$ with the measured ones in a single domain approximation.
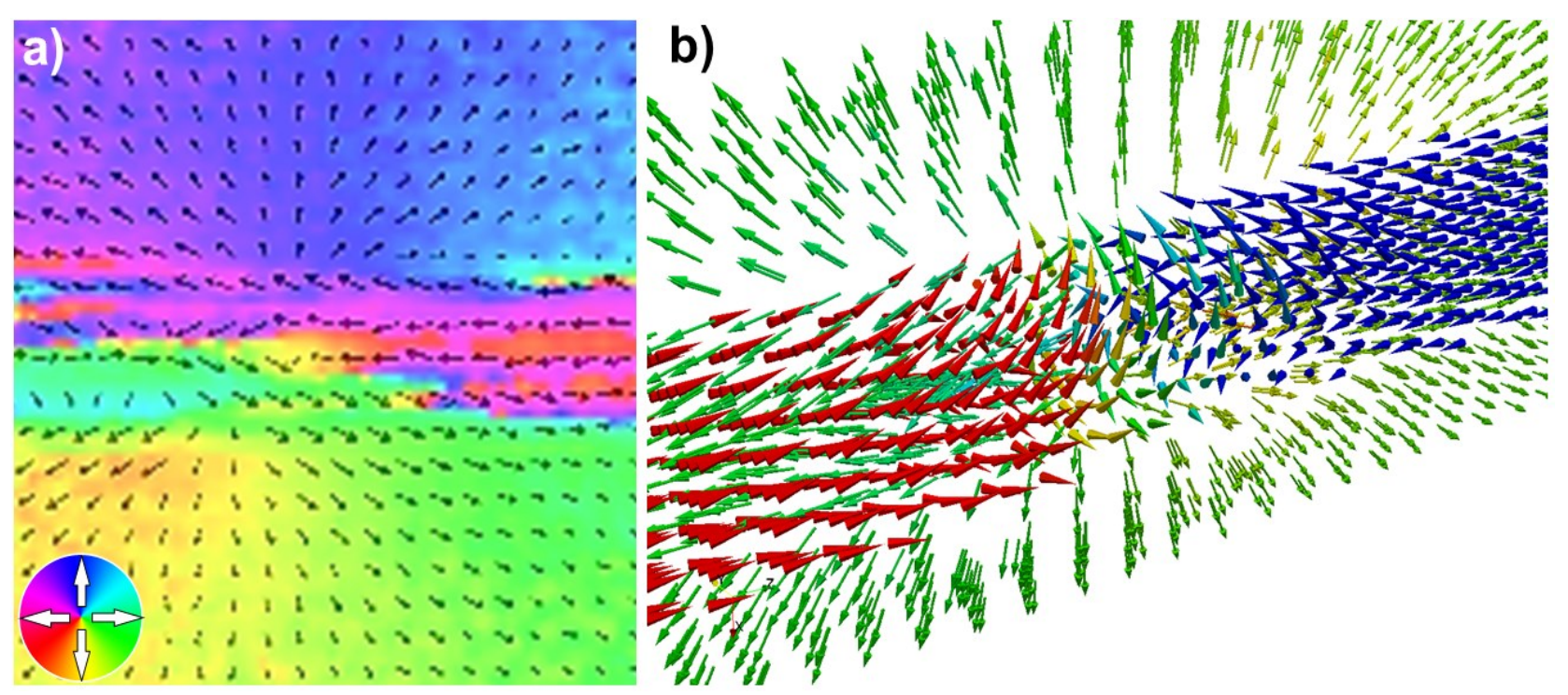

Figure 2. VBF-DPC image and the micromagnetic simulations of the DW in Ni/Co nanowire. a, Color wheel represents the magnetic field direction, arrows show the magnetic field vectors extracted from experimental results. $\mathbf{b}$, The simulation of the vortex domain wall with Bloch point: cones correspond to the component of magnetization in along the $\mathrm{NW}$, arrows shows the stray fields direction. 